\title{
Development of a head-mounted, eye-tracking system for dogs
}

\author{
Abstract \\ Growing interest in canine cognition and visual perception has promoted research \\ into the allocation of visual attention during free-viewing tasks in the dog. The \\ techniques currently available to study this (i.e. preferential looking) have, however, \\ lacked spatial accuracy, permitting only gross judgements of the location of the dog's \\ point of gaze and are limited to a laboratory setting. Here we describe a mobile, head- \\ mounted, video-based, eye-tracking system and a procedure for achieving \\ standardised calibration allowing an output with accuracy of 2-3 .
}

The setup allows free movement of dogs; in addition the procedure does not involve extensive training skills, and is completely non-invasive. This apparatus has the potential to allow the study of gaze patterns in a variety of research applications and could enhance the study of areas such as canine vision, cognition and social interactions.

Keywords: Attention; dogs; eye tracking; gaze; mobility.

\section{Introduction}

Visual exploration of our environment involves a series of saccades (along with head and body movements, which dogs frequently make) to direct our gaze to regions either informative or interesting to us. The preferred regions within a scene are often inspected earlier and attract more fixations and longer viewing time. Gaze patterns hence provide a real-time behaviour index of ongoing perceptual and cognitive 
processing, and could be sensitive indices of our attention, motivation and preference, especially when exploring scenes of high ecological validity (Rayner, 1998; Henderson, 2003; Land et al., 2006).

To monitor human gaze patterns, scleral search coils, dual Purkinje image eye trackers and video-based, pupil-centre/corneal-reflection eye trackers are used in different laboratory settings for different requirements of spatial and temporal resolution. Among these different eye tracking devices, only video-based, headmounted systems allow free head and body movements, and can be used to study naturalistic vision in everyday activities such as driving, playing sports and preparing food (Land et al., 2006).

The adaption of eye-tracking systems for use on non-human animals has, however, resulted in the use of a number of divergent techniques to measure eye movements varying in invasiveness, restraint and level of training required for the use of the apparatus.

With the highest spatial and temporal resolution, scleral search coils are typically employed to study visual processing and eye-movement control in non-human primates, such as macaque monkeys. However the protocol is invasive requiring surgical implantation of a scleral magnetic search coil under the conjunctiva around the eyeball (Judge et al., 1980), which can increase both cost and risk of infection as well as physical discomfort to the animal. An alternative is the use of a video-based remote eye tracker placed close to the animal's head which allows a combination of the pupil and/ or one or more Purkinje image to be tracked, this is typically achieved 
by illuminating the eye using an infrared source, the resulting image being captured by the eye tracker via a camera. Such a technique has been employed with cats (Körding et al., 2001), mice (Stahl et al., 2000), dogs (Jacobs et al., 2006) and macaques (Davis et al., 2009). Whilst this technique offers some methodological refinement by eliminating the need to attach apparatus to the eye, owing to the fixed nature of the cameras it requires the animal's body to be restrained and, often, the head to be fixed, this typically necessitates the surgical insertion of implants into the head which can be attached to external apparatus. Hence whilst developments in head-fixation techniques appear to have reduced the incidence of infection and complications such as bone necrosis (e.g. Davis et al., 2009), the expense, potential stress and possibility of harm to the animal associated with surgery cannot be completely overcome. Not only does fixation of the head and body reduce the opportunities for studying naturalistic behaviour, it also raises concerns over the welfare of the animals used. All scientists working with animals should show a commitment to the 3 R's (Russell and Burch, 1959), and so refinement which improves welfare should be considered important.

The study of the eye movements of freely moving lemurs has been facilitated by the use of a head-mounted, video-based, eye-tracking system adapted from equipment designed for human use (Shepherd and Platt, 2006). This system comprised two small cameras, one recording the visual scene, the other imaging the eye via a reflection from a dichroic mirror. Such a system, however, has currently not been successfully used on non-primate species. 
The dog has long been a model laboratory animal but there is a growing interest in canine cognition in many other contexts. Dogs are of particular interest in the study of social cognition as they are a social species, and therefore likely to be adept at recognising communicative cues. Their history of artificial selection by man and their opportunity for enculturation within the human environment (Virányi et al., 2004) make them a particularly useful model for comparative work with humans.

The study of eye movements and the allocation of visual attention in dogs allows the investigation of factors influencing the human-dog relationship, such as the saliency of human gestures upon dog behaviour (Gácsi et al., 2004; Virányi et al., 2004) and visual processing biases, providing information on putative cognitive mechanisms underlying canine vision (Guo et al., 2009; Racca et al., 2010). Hence, studying gaze patterns has the potential to explore how visual inputs influence a dog's behaviour as well as how these inputs may be processed by the visual system. In addition, it provides a valuable mechanism for studying visual attention itself, both in terms of how it is deployed and also maintained in dogs, a research area that has received little attention. The assessment of looking behaviour in dogs has, however, previously relied largely upon techniques such as measuring the dog's head and body orientation (Gácsi et al., 2004; Virányi et al., 2004) or change of gaze direction (preferential looking) (Guo et al., 2009; Racca et al., 2010). Using either of these methodologies, judgements regarding the allocation of visual attention are restricted in terms of spatial accuracy. Hence whilst these paradigms are useful for assessing variables such as whether the dog oriented their attention towards a stimulus, for example a person, or whether the eyes were attending to the left or right side of a stimulus, they lack spatial accuracy to make more detailed evaluations of the focus of attention. 
Electrooculograms (EOG) have been used to measure cataplexy in narcoleptic dogs (Reid et al., 1996, 1998), however, this necessitates the surgical implantation of electrodes near the orbit of the eye. The EOG signal is also subject to drift, requiring regular recalibrations, this is particularly problematic when working with non-verbal subjects (Aslin and McMurray, 2004). Accuracy of EOG data may be further reduced by nonlinearities (Dell'Osso and Daroff, 1999). In addition, as the EOG technique measures eye movement within the head it is unsuitable for providing point of regard information unless head movement is also measured (Duchowski, 2003). Eye movements in canines have also been studied using infrared reflection tracking systems in conjunction with body stabilisation and a non-invasive, head-fixation technique in order to study the effects of a new surgical treatment for infantile nystagmus syndrome (Dell'Osso et al., 1998, 1999). This methodology has been further advanced by the adaptation of a head-mounted, video-based tracking system, to assess eye movements in dogs with nystagmus (Jacobs et al., 2006). This necessitated mounting the eye cameras on a fixed frame in front of the dog, the dog's body being maintained in a sling with the head manually restrained by an experimenter. Hence, both of these methodologies still limit the range of naturalistic behaviours that can be performed by the subject as well as constraining the proportion of the visual scene which can be viewed.

This paper describes the development and assessment of the accuracy of a headmounted, video-based, eye-tracking system for use on dogs. This equipment is intended to provide a more spatially accurate measure of canine looking behaviour than techniques such as preferential viewing whilst allowing the subject to perform a 
far greater behavioural repertoire than permitted by previous canine eye-tracking methodologies.

\section{Methods}

\subsection{Apparatus}

We adapted a VisionTrak head-mounted eye tracker (ISCAN ETL 500, Polhemus, Vermont, USA) to record gaze patterns from freely moving domestic dogs. The system has a head-mounted eye and scene imager (consisting of a scene camera, an eye camera, an infrared source and a dichroic mirror) which is connected to a host workstation (comprising an RK 826PCI Pupil / Corneal Reflection Tracking Processor and RK 630PCI Autocalibration System) through a cable 4m in length. When used on human participants, this robust eye tracker can collect pupil size, eye movement, and eye point of regard data while allowing complete freedom of head movement. The system has a sampling rate of $60 \mathrm{~Hz}$ and can achieve spatial accuracy up to $0.3^{\circ}$ when used on humans (ISCAN, 2003).

In order to attach a head-mounted eye and scene imager on the dog the apparatus was mounted on an aluminium head strap (Fig. 1). This provided the head strap with rigidity whilst still allowing it to be lightweight and shaped around the dog's head. The strap was attached to the top of a basket muzzle (Baskerville, size 8, The Company of Animals) using a M10 $\times 20 \mathrm{~mm}$ screw with $\mathrm{M} 2 \times 10 \mathrm{~mm}$ screws located either side of it, all secured using nuts. Three screws were used to prevent any lateral movement of the strap. The heads of the screws, which were located inside the muzzle, were countersunk and concealed behind a leather strap, preventing possible discomfort or injury to the dog. The head strap extended along the contour of the 
dog's head, and attached to the muzzle strap at the back of the head by passing underneath it and folding back on top of it. The muzzle strap was held secure within this loop using a M4 $\times 10 \mathrm{~mm}$ screw and nut positioned immediately in front of it in the head strap.

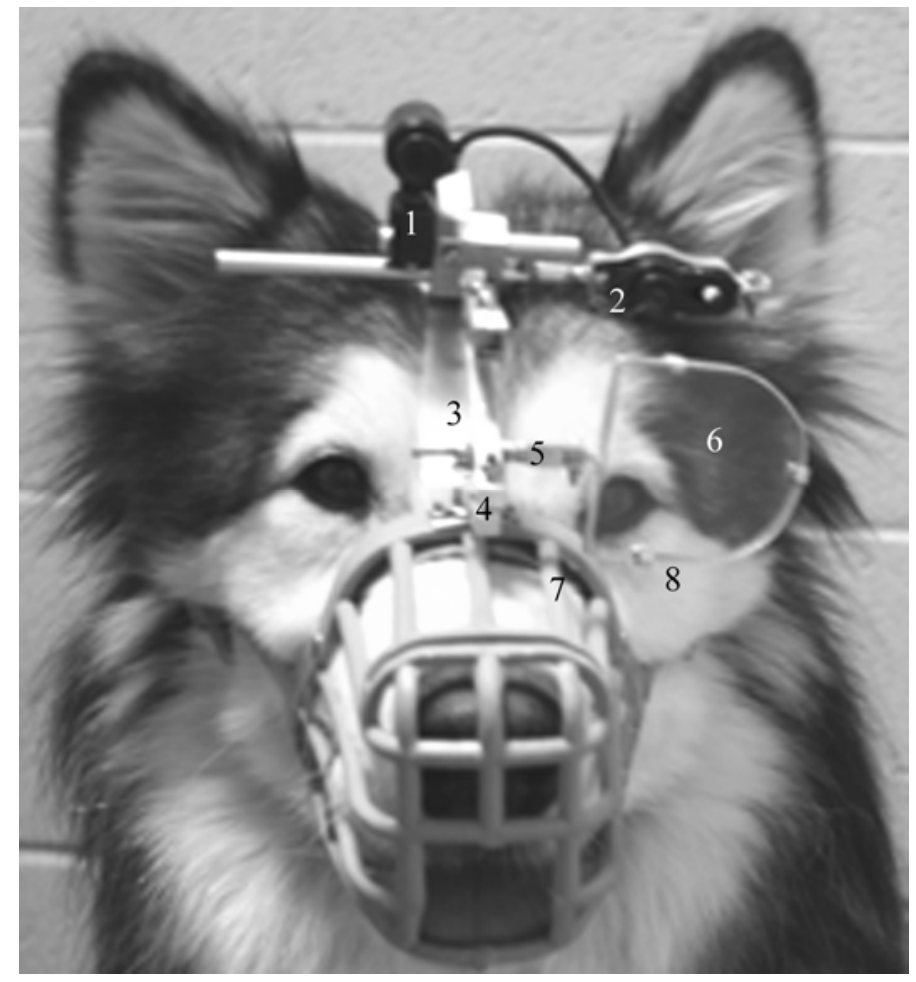

Fig. 1. The eye tracking equipment in place on the dog showing scene camera (1) eye camera (2) head strap (3) mirror support rod (4) mirror clamp (5) dichroic mirror (6) muzzle (7) and mirror frame (8).

Owing to the different head shape of dogs compared to humans it was decided to mount the dichroic mirror in front of the eye rather than below it, as is often the case with head-mounted, eye-tracking systems designed for human use. In addition, due to the diversity of head and eye sizes amongst dog breeds it was necessary to be able to adjust the distance of the mirror from the eye to achieve a clear eye image. To address these issues the mirror was mounted from a square aluminium rod which ran perpendicular to the dog's nose: the mirror support rod. Using a frame and clamp 
device, described below, the mirror could be extended from one of six holes of $2 \mathrm{~mm}$ diameter which were drilled horizontally into the mirror support rod at $7 \mathrm{~mm}$ intervals, with the first positioned $5 \mathrm{~mm}$ from the tip of the rod. Lateral movement of the mirror support rod was inhibited by a second rod, positioned on top of the central nut attaching the head strap to the muzzle, which projected outwards away from the head strap. A piece of threaded rod ran vertically through the end of the mirror support rod, secured by a nut above and below the rod, and located into a hole at the end of this second rod, providing stabilisation.

In order to obtain a clear eye image from a dog with eyes positioned more laterally than those of a human it was necessary for the dichroic mirror to move laterally and also pivot in the horizontal plane, as well as retaining the ability to rotate around the vertical plane, so that it could be positioned parallel to the eye. To facilitate this the mirror was held in an aluminium frame $3 \mathrm{~mm}$ wide and $1 \mathrm{~mm}$ thick which was shaped around its outer edge, with three aluminium supports $15 \mathrm{~mm}$ long and $3 \mathrm{~mm}$ wide located equidistantly around the edge of the frame. The supports were attached to the frame using super glue and curved around either side of the mirror to hold it in place within the frame. Pieces of cushioned adhesive pad were inserted between the supports and the mirror in order to prevent scratching of the dichroic coating. Both of the ends of the strip of metal forming the frame were held in an aluminium block $20 \mathrm{~mm}$ long, $6 \mathrm{~mm}$ wide, $6 \mathrm{~mm}$ deep with a horizontal incision $7 \mathrm{~mm}$ long and $3 \mathrm{~mm}$ wide, the mirror clamp, and secured using a $1 \mathrm{M} \times 13 \mathrm{~mm}$ screw that passed through both the frame and block. This allowed the mirror to be pivoted towards and away from the eye and secured in position by tightening the nut which held the bolt in place. The mirror clamp was threaded onto a piece of threaded rod of $2 \mathrm{~mm}$ diameter 
which passed through the 3rd hole in the mirror support rod and was held in place by two self-locking nuts positioned either side of the mirror support rod, allowing lateral movement and rotation around the vertical plane.

As the optimal eye image was obtained when the eye camera was parallel to the dichroic mirror, the eye camera was also manufactured to move laterally, pivot around the horizontal plane and rotate around the vertical plane. A square aluminium block $20 \mathrm{~mm} \times 20 \mathrm{~mm}$ and $10 \mathrm{~mm}$ deep was mounted on the head strap at the highest position on the dog's head. In front of this was positioned a second aluminium block of the same dimensions into which were drilled 2 holes of $5 \mathrm{~mm}$ diameter, running parallel through the horizontal section of the block and located $10 \mathrm{~mm}$ apart. Two aluminium rods of $5 \mathrm{~mm}$ diameter were positioned in the holes, the higher of these passed into the scene camera casing, the second was $100 \mathrm{~mm}$ long and incorporated a swivel joint which allowed the final $15 \mathrm{~mm}$ to pivot around the horizontal plane. A threaded rod of $2 \mathrm{~mm}$ diameter and $20 \mathrm{~mm}$ length was threaded into this end section of the second rod and passed through a pair of locking nuts which tightened either side of an aluminium frame, $8 \mathrm{~mm}$ wide and $1 \mathrm{~mm}$ thick, shaped around the eye camera to secure it in place. The position of the two camera rods within the block was secured by screws which passed into the front of the block, one located $5 \mathrm{~mm} \times 5 \mathrm{~mm}$ from the top left corner of the block and one $5 \mathrm{~mm} \times 5 \mathrm{~mm}$ from the bottom right corner. When tightened, these screws exerted sufficient pressure on the rods to clamp them in place, permitting the eye camera to be moved laterally as well as rotated in the vertical plane and stabilised in the chosen position. The two aluminium blocks supporting the camera rods were connected via a $1 \mathrm{~mm}$ threaded rod, $28 \mathrm{~mm}$ long, mounted into a 
section of aluminium rod. The threaded rod passed through the centre of the two blocks and screwed into the mirror support rod.

\subsection{Subject}

The subject used for proof of principle was a male Alaskan Malamute, aged two years.

\subsection{Calibration}

The calibration procedure required the dog to visually acquire five points in space, one in each corner of the output captured by the scene camera and one in the centre of the image. In order to ensure standardisation of the calibration procedure the distance between the calibration points and the distance between the dog and the calibration points was fixed. This was achieved using a light metallic cross frame which could be mounted onto the headgear (Fig. 2). The cross consisted of four aluminium rods, $118 \mathrm{~mm}$ long and of $6 \mathrm{~mm}$ diameter each mounted centrally into the sides of a $20 \mathrm{~mm}$ $\times 20 \mathrm{~mm}$ aluminium block, $8 \mathrm{~mm}$ deep. An aluminium rod $6 \mathrm{~mm} \times 6 \mathrm{~mm}$ and $235 \mathrm{~mm}$ long was centrally mounted into one square face of the block. The cross could then be mounted to the mirror support rod in front of the cameras via a $6 \mathrm{~mm} \times 6 \mathrm{~mm}$ aluminium rod $60 \mathrm{~mm}$ long which was tapered at the end to fit smoothly against the slope of the rod. This was attached to the mirror support rod via a $2 \mathrm{M} \times 23 \mathrm{~mm}$ and a $2 \mathrm{M} \times 30 \mathrm{~mm}$ bolt, which ran vertically through both rods. This was connected to a hollow rod $8 \mathrm{~mm} \times 8 \mathrm{~mm}$ and $25 \mathrm{~mm}$ long, via a $2 \mathrm{~mm} \times 20 \mathrm{~mm}$ section of threaded rod which passed vertically through both. The calibration device could then be slotted into this hollow rod and removed once calibration had been achieved. Cardboard squares $25 \mathrm{~mm} \times 25 \mathrm{~mm}$ were attached to the end of each of the four rods forming the 
cross and in front of the central square. This enabled easy identification of the four corner points from the scene output monitor and provided the experimenter with clear targets at which to hold stimuli during the calibration process. This device created a five point calibration system with the four corner calibration points being an equal distance of $25^{\circ}$ away from the central calibration point.

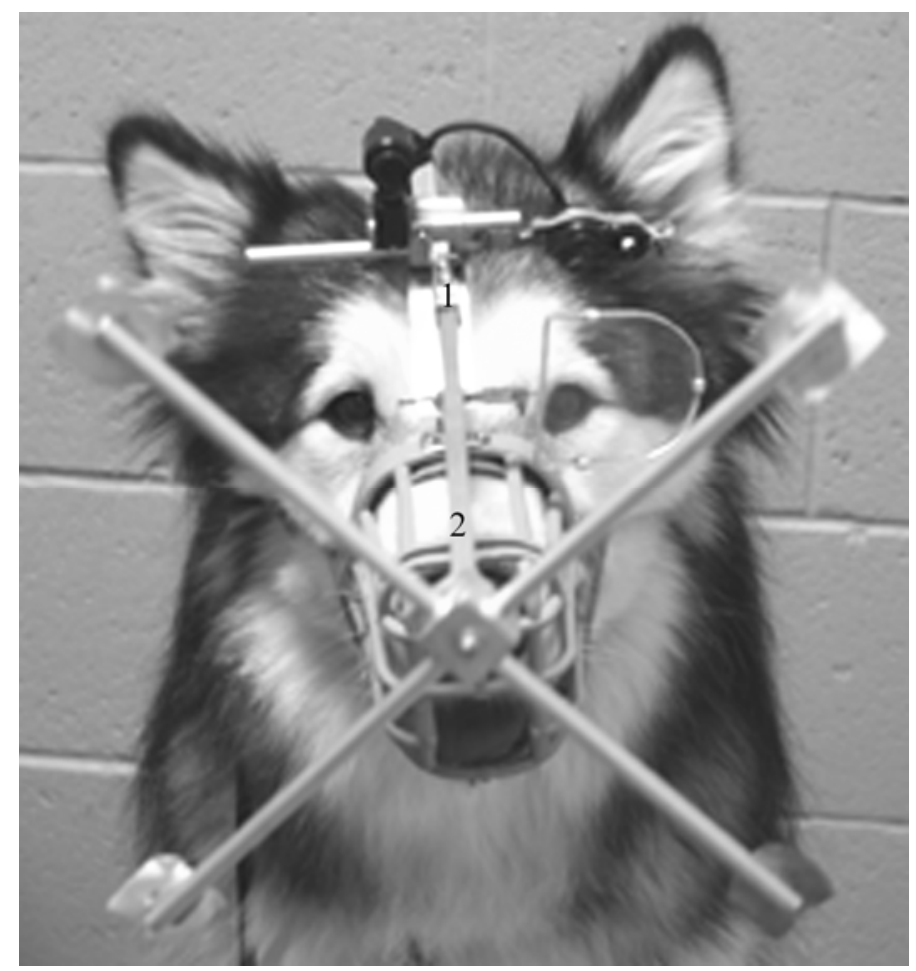

Fig. 2. The calibration cross in place on the head gear showing cross mount (1) and calibration cross (2).

A stimulus, a treat $10 \mathrm{~mm} \times 10 \mathrm{~mm}\left(2.1 \times 2.1^{\circ}\right)$, was held in the centre of each of the five squares in turn, when the dog was judged to be fixating on the treat in any given location the calibration point was entered into the computer. Once all five points had been acquired in this way calibration accuracy could be tested. 


\subsection{Training}

In order to habituate the dog to the apparatus it was initially muzzle-trained using food-based positive reinforcement. Once the muzzle was tolerated for periods up to 30 minutes, the head strap was added and the habituation procedure repeated, this process was repeated twice more for the addition of the cameras and the mirror. In order to carry out calibration the dog was trained to visually track a treat using eye movements with minimal movement of the head. This was achieved using positive reinforcement; an audible click was used to mark the desired behaviour more precisely. Initially, visual following of a treat was accompanied by large head movements, however, over approximately 10 training sessions head movements became minimal and eye movements increased. The same methods were used to train the dog to maintain fixation on the treat when it was held stationary. The dog was then gradually habituated to the calibration cross being slotted into the front of the headgear over approximately 5 training sessions, the cross was initially tolerated for 10-20 seconds without behaviours which attempted to remove it, such as pawing at the device, this was increased to 2-3 minutes during training. Following this the dog was reinforced for fixating on treats held at the five calibration point locations.

\subsection{Data collection}

The eye tracker was calibrated as described in section 2.3. The treat was held at each of the calibration point locations in such a way that the experimenter's hand and arm did not enter the space between the five points in order to prevent this acting as a distraction. When attracting the dog's attention towards the treat the experimenter called the dog's name and pointed to it. If the dog continued not to look at the treat it was removed from its position and then replaced. For accuracy, only footage with no 
movement or vocalisation was coded. The order in which the treat was held at the five locations was randomised between trials in order to prevent learned behaviour influencing the results. No rewards were given during the trial; once the trial had ended the dog received the treat. All output from the eye tracker was recorded onto DVD via a Logik LDVR808 DVD recorder. Nineteen trials were conducted of which five provided codeable data for all five calibration points.

\subsection{Treatment of data}

Output from the eye tracker was coded frame by frame at a rate of 30 frames per second, using a DVD player and a 290mm $\times 230 \mathrm{~mm}$ Philips LDH2114\10 video monitor. For each of the five calibration points the distance was measured between the centre of the treat when held in position and the centre of the output crosshair in a direct line. The vertical and horizontal distance between the output crosshair and the stimulus was also measured. The visual angle between the treat and the crosshair was calculated for each of these measurements on a frame-by-frame basis.

As previous information concerning the use of eye tracking technology on dogs is extremely limited there is a lack of data regarding the features of fixations in this species during visual exploration. Therefore two parameters were examined in detail: first fixation (the first set of data which matched the fixation criteria) and closest fixation (the dataset matching the criteria in which the output crosshair was closest to the fixation point). These two sets of data were considered as eye-tracking research in humans has demonstrated that first fixations on a stimulus tend to be less accurate than subsequent fixations as they can be subject to overshoot and undershoot (Bötzel et al., 1993). To qualify as a fixation the output crosshair had to fall within $30 \mathrm{~mm}$ 
$\left(5.8^{\circ}\right)$ of the fixation point (centre of the treat) and not move around the fixation point at an average speed of more than $25 \%$ second for a given duration. The data were examined using five different minimum durations for this behaviour. These were 67, $100,133,167$ or $200 \mathrm{~ms} .30 \mathrm{~mm}\left(5.8^{\circ}\right)$ was chosen as the maximum distance that the output crosshair could fall from the fixation point, since the minimum distance between any two fixation points on the metal calibration cross was $120 \mathrm{~mm}\left(25^{\circ}\right)$ (i.e. distance from centre of the cross to the centre of the cardboard squares positioned at the end of the arms). Thus the distance between any two fixation points was at least twice the radius around them (Fig. 3).

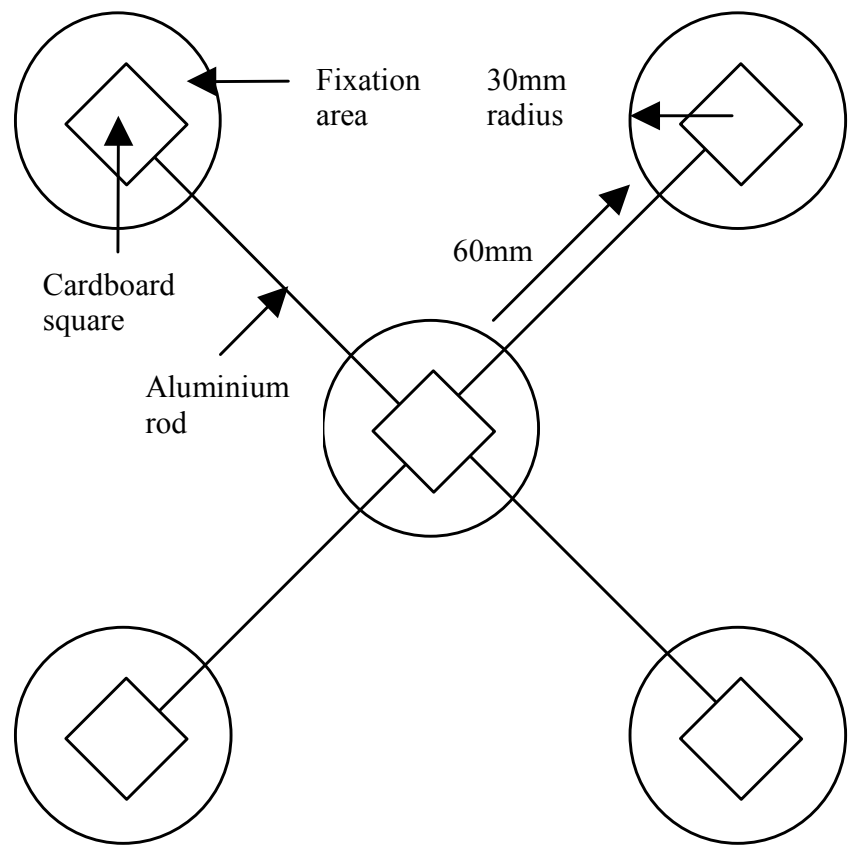


Fig. 3. Scale diagram of calibration equipment and fixation classification areas. Scale 1:3.

Accuracy was assessed from the mean distance that the centre of the crosshair remained from the centre of the fixation point during a fixation. Data for each of the five calibration points were averaged across the five trials, providing an overall accuracy level for each point. Accuracy for first and closest fixations was obtained for each of the fixation durations; these were then averaged across all durations to calculate the overall accuracy of the system for first and closest fixations. Horizontal and vertical accuracy between the crosshair and the fixation point was calculated for each fixation in the same manner.

\subsection{Statistical Analysis}

Initial summary descriptive statistics were calculated for accuracy based on mean deviation from centre of fixation point. Accuracy for first versus closest fixation for different fixation durations was compared using a repeated measures ANOVA. In order to examine the relationship between accuracy and fixation length for the closest fixation data, regression analysis using a quadratic equation was used following inspection of its graphical representation. Evaluation of these results was used to determine the recommended optimal fixation duration to use in practice. 


\section{Results}

The overall accuracy using different fixation duration criteria was around $3^{\circ}$ (Table $1)$.

\begin{tabular}{lll}
$\begin{array}{l}\text { Minimum length } \\
\text { of fixation }(\mathrm{ms})\end{array}$ & $\begin{array}{l}\text { Accuracy }\left(^{\circ}\right) \\
\text { first fixation }\end{array}$ & Accuracy $\left(^{\circ}\right)$ \\
& Mean \pm SD & Mean \pm SD \\
\hline 67 & $3.76 \pm 0.58$ & $2.25 \pm 0.47$ \\
100 & $3.69 \pm 0.73$ & $2.51 \pm 0.6$ \\
133 & $3.48 \pm 0.81$ & $2.6 \pm 0.58$ \\
167 & $3.62 \pm 1.06$ & $2.67 \pm 0.62$ \\
200 & $3.41 \pm 1.09$ & $2.71 \pm 0.67$ \\
\hline
\end{tabular}

Table 1: Accuracy of crosshair location on video output in relation to predetermined focal points for fixations defined by varying durations. First fixation $=$ first dataset matching fixation criteria, closest fixation $=$ dataset matching fixation criteria during which output crosshair was closest to defined focal point.

The mean level of accuracy for the first fixation across all durations was $3.59^{\circ} \pm 0.07$ (radius $\pm \mathrm{SEM}$ ). The mean level of accuracy for the closest fixation was $2.55^{\circ} \pm 0.08$. Closest fixation produced a higher level of accuracy (repeated measures ANOVA: $\left.\mathrm{F}(1,4)=55.93 \mathrm{p}=0.002 \operatorname{partial} \eta^{2}=0.93\right)$. 
The mean vertical and horizontal accuracy for the first fixations across all durations were $2.31^{\circ} \pm 0.09$ and $2.53^{\circ} \pm 0.04$, respectively. The mean levels of vertical and horizontal accuracy for the closest fixations were $1.63^{\circ} \pm 0.03$ and $1.85^{\circ} \pm 0.07$. Vertical accuracy was greater than horizontal accuracy for the closest fixations (repeated measures ANOVA: $F(1,4)=31.390 p=0.005$ partial $\eta^{2}=0.89$ ). No significant differences were found between vertical and horizontal accuracy for the first fixations. Horizontal and vertical accuracy across all fixation durations for the closest durations are shown in Table 2.

$\begin{array}{lll}\text { Minimum length } & \text { Vertical } & \text { Horizontal } \\ \text { of fixation }(\mathrm{ms}) & \text { accuracy }\left({ }^{\circ}\right) & \operatorname{accuracy}\left({ }^{\circ}\right)\end{array}$

67

$1.51 \pm 0.33$

$1.66 \pm 0.31$

100

$1.61 \pm 0.3$

$1.74 \pm 0.44$

133

$1.65 \pm 0.4$

$1.85 \pm 0.33$

167

$1.68 \pm 0.38$

$1.95 \pm 0.41$

200

$1.69 \pm 0.4$

$2.03 \pm 0.52$

Table 2: Vertical and horizontal accuracy of crosshair location on video output for closest fixations defined by varying durations. Values presented in the table are Mean \pm SD

Regression analysis using a quadratic equation produced a model with excellent fit (adjusted R squared $=96.4 \%$ ) to describe the relationship between accuracy and the 
duration used to define a fixation $($ accuracy $=1.648+0.01125$ fixation -0.00003 fixation $^{2}$ ). Visual inspection of the data, suggests that the point of maximum inflection of the curve occurs around 100ms (Fig. 4) and that accuracy stabilises beyond 200ms. Since the apparent accuracy will be affected by both the number of frames sampled as well as the reliability of the system per se, the optimal fixation duration to use in practice is based upon consideration of both the relationship between accuracy and fixation duration as well as the point at which this relationship stabilises. On this basis we suggest that $100 \mathrm{~ms}$ represents the minimum optimal fixation length to use in practice, since below this time, the apparently high level of accuracy may be an artefact of the limited data used in its determination.

Data for the accuracy of individual fixation points using the closest fixation criterion are given in Table 3 .

\begin{tabular}{|c|c|c|}
\hline \multirow[t]{3}{*}{ Calibration point } & Accuracy $\left(^{\circ}\right)$ minimum & Accuracy $\left(^{\circ}\right)$ minimum \\
\hline & fixation $100 \mathrm{~ms}$ & fixation $200 \mathrm{~ms}$ \\
\hline & Mean \pm SD & Mean \pm SD \\
\hline
\end{tabular}

Top left $\quad 2.84 \pm 1.46 \quad 2.84 \pm 1.46$




\begin{tabular}{lcc} 
Top right & $2.84 \pm 1.74$ & $3.48 \pm 2.31$ \\
Bottom left & $2.00 \pm 0.80$ & $2.32 \pm 0.89$ \\
Bottom right & $1.75 \pm 0.48$ & $1.77 \pm 0.51$ \\
Centre & $3.12 \pm 1.19$ & $3.12 \pm 1.19$ \\
\hline
\end{tabular}

Table 3: Output accuracy levels for individual calibration points

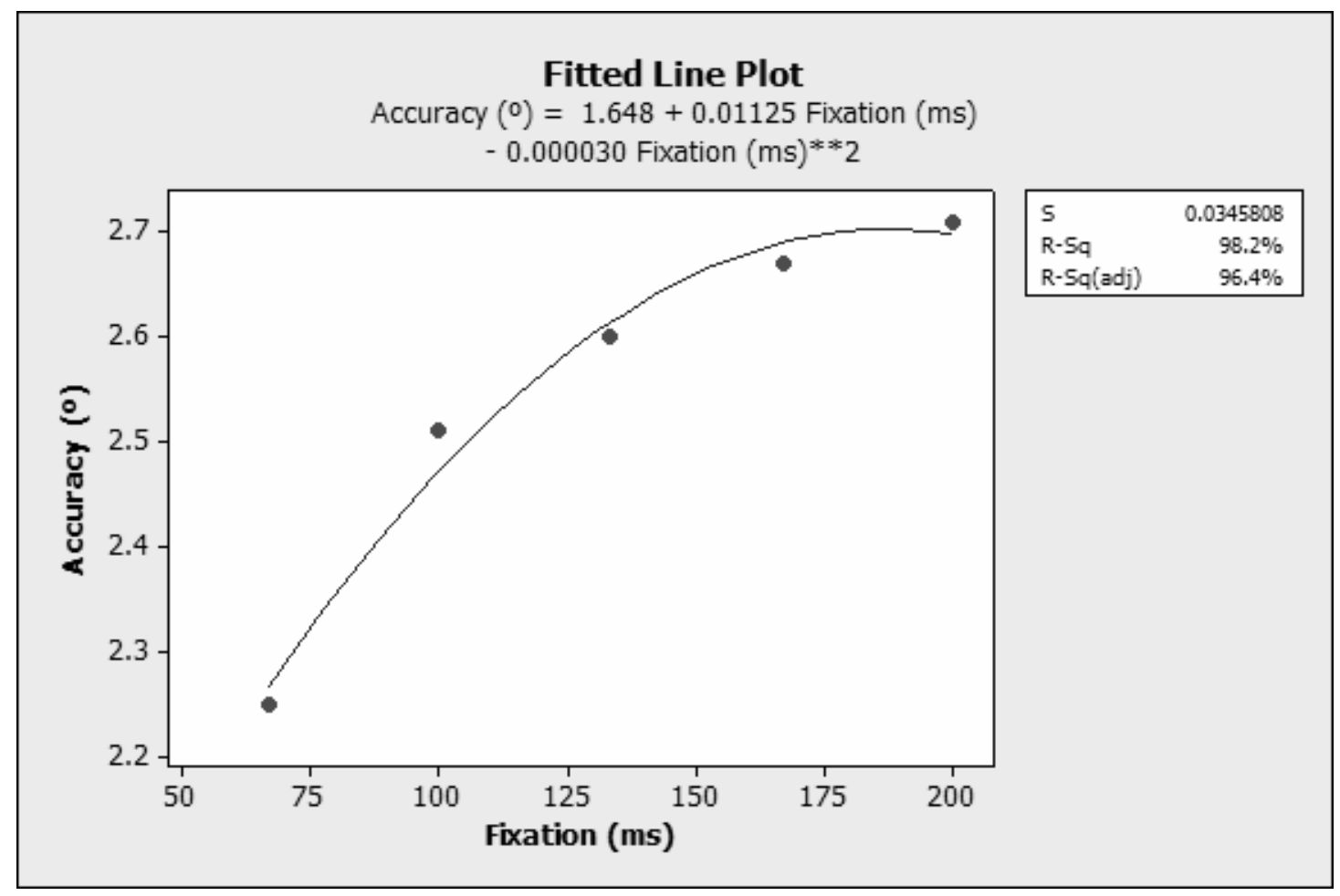

Fig. 4. Accuracy $\left({ }^{\circ}\right)$ versus minimum fixation lengths for closest fixations with fitted line. Increase in degrees indicates reduced accuracy.

It is suggested that the following definition be applied in future when using this equipment to determine fixations by the dog: a period of at least $100 \mathrm{~ms}$ duration during which the output crosshair falls within $6^{\circ}$ of a region of interest and does not 
move around the centre of the point of interest at an average speed of more than $25^{\circ} /$ second. For calibration purposes it is suggested that the closest fixation be used.

\section{Discussion}

We have developed and described a reliable head-mounted, eye-tracking system suitable for use on dogs and a standardised calibration procedure for this equipment. The accuracy level of $2.25-2.71^{\circ}$ achieved using this system is within the bounds of upper accuracy levels obtained recording the eye movements of other non-human species (Shepherd and Platt, 1996; Guo et al, 2003). We have also provided a suggested definition of a fixation for future research utilising this apparatus on dogs. Our mean vertical accuracy of $1.63^{\circ}$ for closest fixations is very consistent with a canine area centralis which extends $\pm 1.5^{\circ}$ vertically (Jacobs et al., 2006). Greater discrepancy in horizontal accuracy between the current finding of $1.85^{\circ}$ and a reported area centralis extending $\pm 3^{\circ}$ (Jacobs et al., 2006) may reflect variation in the distribution of retinal ganglion cells that has been noted between dogs with different nose lengths (McGreevy et al., 2006). In addition, our finding that first fixations were less accurate than subsequent fixations is consistent with dogs employing similar eye movements to humans when making saccades towards and fixations upon a stimulus in which the eye positions itself with greater accuracy during subsequent fixations. This also offers a possible explanation for our finding of a significant difference between vertical and horizontal accuracy for closest but not first fixations, as during the first fixation the stimulus may not be positioned on the area centralis of the retina.

Use of a video-based, eye-tracking system on a non-human species without head restraint does pose some logistical challenges. In particular, the amount of training 
required to habituate the subject to the equipment and to teach the animal to follow and fixate on a stimulus with minimal head movements in order to achieve accurate calibration. However, even though the training may be time consuming, it is relatively straightforward, being based on simple habituation and routinely used positive-reward operant procedures. A further potential problem is the introduction of error into the accuracy of data due to minor movements of the equipment on the dog's head during testing. For this reason it is suggested that output accuracy is assessed immediately following calibration, using the method described in section 2.5, and again at the end of each testing block. Restricting the length of testing sessions to time periods for which the dog will tolerate the equipment without becoming restless would also help to overcome this issue. As calibration is performed with the dog facing the experimenter the direction of gaze can also be visually assessed as the calibration points are entered into the system.

The current study utilised a narrow-view scene camera lens intended for studying close-range dog-dog and dog-human interactions, adaptations to the equipment and calibration protocol may be necessary when the wide-view scene camera is used for studying dog's gaze behaviour towards visual stimuli at greater distances. As the viewing distance of our calibration grid (approximately $30 \mathrm{~cm}$ ) is very close to dogs' optimal accommodation range (33-50cm; Miller and Murphy, 1995), it is unlikely our calibration distance will induce near point of convergence in dogs. Furthermore, we use dogs with reasonable binocular vision in our research as this reduces the possibility of convergence, due to the need to maintain binocular vision, during the calibration procedure. In addition, this measure will facilitate the comparison of acquired data with that from humans and non-human primates. 
Use of a monocular eye tracker can have limitations and present challenges.

Information regarding the intersection of gaze angles from both eyes is not available to facilitate the identification of targets at depths different to that for which the equipment has been calibrated. Care must also be taken when selecting appropriate subjects, for example, achiasmatic dogs have been shown to display monocular saccades (Dell'Osso and Williams, 1995) and hence representative eye movement data could not be obtained from such animals using a monocular device. In addition, dogs in general may show looser yoking of the two eyes than is found in humans and non-human primates (Dell'Osso and Williams, 1995). If this is the case then the current methodology of conducting a binocular calibration, with both eyes viewing the stimuli, may result in a less accurate output than monocular calibration, in which only the eye being tracked would view the calibration stimulus, ensuring that stimuli are fixated by the correct eye. Binocular calibration was employed in the current study as it can be performed more quickly, is less intrusive and requires less training than monocular calibration. In addition, in some circumstances, such as our intention to study gaze behaviour in naturalistic settings, dogs use both eyes to view scenes. Therefore, given that monocular calibration with a monocular eye-tracker would only allow one eye to view the calibration points, binocular calibration may be more ecologically valid and more comparable with data obtained from a dog viewing binocularly. The protocol of binocular calibration with a monocular eye-tracking system is not uncommon in studies of fixational and scene viewing gaze behaviour in humans and non-human primates (e.g. Guo et al., 2003; Guo, 2007; Steckenfinger and Ghazanfar, 2009; Shepherd et al., 2010). Previous studies have revealed that during a saccade, the eyes may initially diverge, but convergence occurs in the later part of the 
saccade, continuing into the following fixation period (e.g. Collewijn et al., 1995), suggesting that maximum disparity between the eyes occurs during saccades with increased convergence during fixations. Indeed, it has been suggested that for tasks that do not require far distance or depth perception, such as reading, monocular and binocular calibration produce comparable results in human adults (Nuthmann and Kliegl, 2009). Given the relative infancy of canine eye-tracking, particularly using monocular equipment, there is a lack of information regarding the effects of monocular and binocular calibration; further research in this area is necessary to inform future calibration protocols.

Despite these caveats the monocular system employed here provides a number of benefits for conducting behavioural research with dogs. It is unobtrusive; the only equipment placed in front of the eye is the transparent mirror and the lack of restraint required may permit longer recording sessions than would be tolerated by a restrained subject. The equipment is relatively inexpensive and can be easily operated by a single experimenter. In addition, calibration can be conducted quickly, which is highly beneficial when working with non-verbal subjects. Monocular systems have been commonly used to study natural vision in humans navigating through the environment (Cheong et al., 2008) and when looking at more detailed stimuli such as faces (Kleinhans et al., 2008), as well as in non-human animals (Shepherd and Platt, 2006).

The five calibration point locations, the centre and four corners of the scene output, were chosen to assess the accuracy of the system as these positions are typically used for rapid calibration in head-mounted eye trackers. Use of these positions may be 
more susceptible to cross-talk errors between the horizontal and vertical axes compared to points actually located on the two axes (i.e. left, right, top and bottom). However, overall, the use of these corner points is likely to allow a more naturalistic account of eye movements by considering non-linear interactions between the horizontal and vertical planes.

Our system appears to provide a greater level of accuracy than the head-mounted ISCAN system used with macaques by Shepard and Platt (2006), which achieved an accuracy of $5-10^{\circ}$. This may reflect the more standardised calibration procedure employed in the current study. The calibration cross enabled standardisation of the distance between both the calibration points and the subject and the distance between the points themselves, whilst Shepherd and Platt (2006) calibrated their equipment with a trainer holding treats entering the visual scene and standing at the five calibration locations, permitting less standardisation of distances. Whilst the current technique provides less spatial accuracy than that provided by the scleral search coil technique employed by Guo et al. (2003) in primates, which achieved an accuracy level under $1^{\circ}$, the current technique permits investigation in more naturalistic settings, allowing movement of both head and body, as well as avoiding the surgery required to implant a coil into the eye of a non-human species. By allowing a greater behavioural repertoire including head movement, a larger proportion of the visual scene can be scanned by a subject wearing the current device compared to that used in other techniques such as that employed by Jacobs et al (2006), allowing a more enriched view of visual attention. Hence, whilst not suitable for recording very detailed eye movement information, it is hoped that this equipment will permit a 
naturalistic method for studying the allocation of dogs' visual attention in nature vision.

In future, the authors hope to use this system to study dogs' visual attention in a variety of naturalistic settings, such as in social interactions both with members of their own species and humans. It also has the potential to provide a measure of visual acuity both between dog breeds and in specific individuals. As allocation of visual attention can be used to assess cognitive function (Crutcher et al., 2009), the system could also be applied to the identification of cognitive changes and degeneration.

In conclusion, this system provides a non-invasive method of assessing dogs' looking behaviour without restraint with a higher level of spatial accuracy than previously available for this species, and has potential application in a wide variety of research settings.

\section{Acknowledgements}

The authors wish to thank David Williams for his help and guidance with manufacture of the equipment, Paul Coster, George Rodis and Andrew Sherman for their technical support and Petronella Nilsson for her assistance during the training process. 


\section{References:}

Aslin RN, McMurray B. Automated corneal-reflection eye tracking in infancy: methodological developments and applications to cognition. Infancy, 2004;6:155-63.

Bötzel K, Rottach K, Büttner U. Normal and pathological saccadic dysmetria. Brain, 1993;116:337-53.

Cheong AMY, Geruschat DR, Congdon N. Traffic gap judgment in people with significant peripheral field loss. Optometry Vision Sci., 2008; 85:26-36.

Collewijn H, Erkelens CJ, Steinman RM. Voluntary binocular gaze-shifts in the plane of regard: dynamics of version and vergence. Vision Res., 1995;35:3335-58.

Crutcher MD, Calhoun-Haney R, Manzanares CM, Lah JJ, Zola SM. Eye tracking during a visual paired comparison task as a predictor of early dementia. Am J Alzheimer's Dis., 2009;24:258-66

Davis TS, Torab K, House P, Greger B. A minimally invasive approach to long term head fixation in behaving nonhuman primates. J Neurosci Meth., 2009;181:106-10. Dell'osso LF, Daroff RB. Eye movement characteristics and recording techniques. In Glaser JS, editor. Neuro-Ophthalmology. Lippincott, Williams \& Wilkins: Philadelphia, 1999:327-43.

Dell'Osso LF, Hertle RW, Williams RW, Jacobs JB. A new surgery for congenital nystagmus: effects of tenotomy on an achiasmatic canine and the role of extraocular proprioception. JAAPOS, 1999;3:166-82.

Dell'Osso LF, Williams RW. Ocular motor abnormalities in achiasmatic mutant Belgian sheepdogs: unyoked eye movements in a mammal. Vision Res., 1995;35:109-16. 
Dell'Osso LF, Williams RW, Jacobs JB, Erchul DM. The congenital and seesaw nystagmus in the prototypical achiasma of canines: comparison to the human achiasmatic prototype. Vision Res., 1998;38:1629-41.

Duchowski AT. Eye Tracking Methodology: Theory and Practice, first ed. Springer: Berlin, 2003: 56.

Gácsi M, Miklósi A, Varga O, Topál J, Csányi V. Are readers of our face readers of our minds? Dogs (Canis familiaris) show situation dependent recognition of humans' attention. Anim Cogn., 2004;7:144-53

Guo K. Initial fixation placement in face images is driven by top-down guidance. Exp Brain Res., 2007;181:673-77.

Guo K, Meints K, Hall C, Hall S, Mills D. Left gaze bias in humans, rhesus monkeys and domestic dogs. Anim Cogn., 2009;12:409-18.

Guo K, Robertson RG, Mahmoodi S, Tadmor Y, Young MP. How do monkeys view faces? - A study of eye movements. Exp Brain Res., 2003;150: 363-74.

Henderson JM. Human gaze control during real world scene perception. Trends Cogn Sci., 2003;7:498-504.

ISCAN Inc. ISCAN ETL-500 Operating Instructions. ISCAN Inc.: Woburn, 2003:3. Jacobs JB, Dell'Osso LF, Hertle RW, Acland GM, Bennett J. Eye movement recordings as an effectiveness indicator of gene therapy in RPE65 deficient canines: implications for the ocular motor system. Invest Opthalmol Vis Sci., 2006;47:286575.

Judge SJ, Richmond BJ, Chu FC. Implantation of magnetic search coils for measurement of eye position: an improved method. Vision Res., 1980;20:535-38. 
Kleinhans NM, Richards T, Stirling L, Stegbauer KC, Mahurin R, Johnson LC, Greenson J, Dawson G, Aylward E. Abnormal functional connectivity in autism spectrum disorders during face processing. Brain, 2008; 131:1000-12.

Körding KP, Kayser C, Betsch BY, König P. Non contact eye tracking on cats. J Neurosci Meth., 2001;110:103-11.

Land MF. Eye movements and the control of actions in everyday life. Prog Retin Eye Res., 2006;25:296-334.

McGreevy PD, Grassi TD, Harman AM. A strong correlation exists between the distribution of retinal ganglion cells and nose length in the dog. Brain Behav Evolut., 2004;63:13-22.

Miller PE, Murphy CJ. Vision in Dogs. J Am Vet Med Assoc., 1995;207:1623-34.

Nuthmann A, Kliegl R. An examination of binocular reading fixations based on sentence corpus data. J Vision, 2009;9:1-28.

Racca A, Amadei E, Ligout S, Guo K, Meints K, Mills D. Discrimination of human and dog faces and inversion responses in domestic dogs (Canis familiaris). Anim Cogn., 2010;13:525-33.

Rayner K. Eye movements in reading and information processing. Psychol Bull., $1998 ; 124: 372-422$.

Reid MS, Nishino S, Tafti M, Siegel JM, Dement WC, Mignot E.

Neuropharmacological characterisation of basal forebrain cholinergic stimulated cataplexy in narcoleptic canines. Exp Neurol., 1998;151:89-104.

Reid MS, Tafti M, Nishino S, Sampathkumaran R, Siegel JM, Mignot E. Local administration of dopaminergic drugs into the ventral tegmental area modulates cataplexy in the narcoleptic canine. Brain Res., 1996;733:83-100. 
Russell WMS, Burch RL. The Principles of Humane Experimental Technique, first ed. Methuen: London, 1959.

Shepherd SV, Platt ML. Noninvasive telemetric gaze tracking in freely moving socially housed prosimian primates. Methods, 2006;38:185-94.

Shepherd SV, Steckenfinger SA, Hasson U, Ghazanfar AA. Human-monkey gaze correlations reveal convergent and divergent patterns of movie viewing. Curr Biol., 2010;20:649-56.

Stahl JS, van Alphen AM, De Zeeuw CI. A comparison of video and magnetic search coil recordings of mouse eye movements. J Neurosci Meth., 2000;99:101-10.

Steckenfinger SA, Ghazanfar AA. Monkey visual behavior falls into the uncanny valley. P Natl Acad Sci USA, 2009;106:18362-466.

Virányi Z, Topál J, Gácsi M, Miklósi A, Csányi V. Dogs respond appropriately to cues of humans' attentional focus. Behav Process., 2004;66:161-72. 